\title{
Opiniões sobre a doença entre membros da rede social de pacientes de hanseníase no Recife ${ }^{1}$
}

\author{
Katia V. de O. Feliciano ${ }^{2}$ e Maria Helena Kovacs ${ }^{2}$
}

RESUMO O presente artigo descreve um estudo realizado no Recife, Brasil, entre novembro de 1993 e julho de 1994, com a finalidade de explorar as opiniões dos membros da rede social (por exemplo, familiares, amigos e vizinhos) de portadores de hanseníase na avaliação, interpretação e manejo das manifestações corporais da doença na trajetória que levou ao diagnóstico. A amostra constou de 93 membros da rede social, com idade entre 20 e 70 anos, que apoiaram o curso de ação de 83 pacientes diagnosticados no período do estudo. A análise buscou detectar variáveis capazes de discriminar os membros da rede de apoio dos pacientes de hanseníase classificados como casos (presença de incapacidades ou lesões precursoras de incapacidades) ou controles.

$O$ estudo evidenciou a escassez de informações sobre a transmissão da hanseníase e revelou um quadro transicional onde se confrontam expectativa de cura e uma visão estigmatizante das conseqüências da doença. Apenas uma quarta parte dos sujeitos do estudo suspeitou, antes do diagnóstico, que o paciente era portador de hanseníase, o que sugere baixa percepção do risco representado pela doença e reforça a concepção da invisibilidade das suas manifestações corporais. Os resultados mostram um perfil de percepção e manejo da hanseníase que pode facilitar a propagação da doença e a instalação ou agravamento das suas conseqüências físicas e sociais.

A combinação de limitação funcional, preconceito social e sofrimento humano que acompanham a hanseníase explica por que essa doença é mais temida do que outras doenças, inclusive doenças fatais. Em vários contextos a imagem social negativa da hanseníase afeta a avaliação e o manejo dos danos e da disponibilidade de medidas eficazes para a sua prevenção e

\footnotetext{
Investigação financiada pelo Programa Especial para Pesquisa e Treinamento em Doenças Tropicais (TDR) (Projeto ID-920523), Programa das Nações Unidas para o Desenvolvimento/Banco Mundial/ Organização Mundial da Saúde.

2 Faculdade de Ciências Médicas de Pernambuco, FESP/UPE, Rua Arnóbio Marques, 310, Santo Amaro, Recife, PE, CEP 50100-130, Brasil. Fax 55-081-4233371.
}

tratamento, com evidente reflexo na efetividade das ações desenvolvidas a fim de conseguir sua eliminação.

Estudos (1-3) têm demonstrado que as ações cujo alvo é o controle ou erradicação de uma doença devem estar atentas à percepção, avaliação e manejo da doença em questão pelos grupos a quem a ação se dirige-uma vez que existem diferenças significativas nas formas de perceber e agir apresentadas pelo doente, sua rede social de referência e pelos profissionais de saúde.

A rede social de apoio, formada pelo conjunto de relações entre pessoas (familiares, amigos, vizinhos, etc.) que fornecem ajuda para que o indivíduo enfrente as situações do cotidiano (4), representa uma referência importante para o paciente de hanseníase por guardar íntima relação com os seus valores e normas. Esta relação tem implicações-como o grau de atenção à saúde-evidentes para o funcionamento de sistemas sociais (5-9). O conteúdo das relações entre membros da rede social responde às necessidades geradas pelas circunstâncias da vida diária dos sujeitos envolvidos. A estrutura desta rede resulta, por exemplo, dos compromissos familiares, da disponibilidade para ajuda, da confiança e conhecimento do outro. Existe, em geral, uma opção para selecionar os participantes segundo as preferências, as necessidades ou as obrigações recíprocas $(4,5)$. 
A rede social mobiliza recursos financeiros, materiais e emocionais, compartilha tarefas e informações e funciona como um sistema de apoio importante na definição de problemas, mediando as normas culturais e atribuindo significado aos acontecimentos, influenciando a opção por alternativas de ação e avaliando suas conveniências práticas. Nos microprocessos que envolvem a influência dos grupos sociais de referência nas decisões para a busca de ajuda e uso dos recursos de saúde, os sujeitos se afetam mutuamente e se inter-relacionam com o complexo social global $(5,10)$.

A forma como a hanseníase é incorporada na construção da realidade familiar (11) e as mudanças que traz para a vida do paciente e dos membros da sua família estão relacionadas com o funcionamento do ciclo vital familiar e com o significado atribuído às experiências prévias com problemas de saúde em geral e com a hanseníase em particular. Essas experiências são vivenciadas no cotidiano de segmentos sociais distintos que apresentam não apenas um perfil epidemiológico diferente, mas um pensar, sentir e agir diversos em relação às questões da saúde $(3,12)$.

Na prática comunicativa cotidiana, este saber é cristalizado na rede de interações de grupos sociais na forma de valores e normas (13). Portanto, no processo de avaliação e tomada de decisão para o manejo da doença, os significados compartilhados são negociados entre indivíduos que tentam persuadir outros sobre a intensidade dos problemas de saúde e a necessidade de buscar mais recursos, ou que tentam atenuar as implicações da doença. A investigação da relação entre o paciente de hanseníase e sua rede social como instrumento para eliminação da doença se justifica na medida em que esta relação se constitui em um dos fatores envolvidos na persistência da transmissibilidade da hanseníase, no desenvolvimento das incapacidades por ela provocadas e na manutenção do preconceito contra os seus portadores.

O presente estudo buscou identificar as opiniões desenvolvidas pelos membros da rede social dos portadores de hanseníase no processo de avaliação, interpretação e manejo das manifestações corporais da doença. Ademais, buscou explorar a influência destas opiniões na trajetória que conduziu ao diagnóstico. As principais limitações deste trabalho são o seu caráter retrospectivo e a inclusão apenas dos membros da rede social de pacientes que tiveram acesso aos serviços de saúde. Por outro lado, é fundamental considerar que esta investigação representa uma aproximação preliminar a uma realidade de caráter complexo que merece estudos mais detalhados.

\section{MATERIAIS E MÉTODOS}

Este estudo descritivo, de caráter exploratório, é parte de uma pesquisa mais ampla, realizada de novembro de 1993 a julho de 1994 no município do Recife, da qual constaram, ademais: a) um estudo de caso-controle efetuado para compreender a influência da percepção e interpretação desenvolvidas por portadores de hanseníase no manejo da doença e no uso dos serviços de saúde e b) uma avaliação das condições técnicas e operacionais existentes na rede básica de saúde para o diagnóstico da hanseníase. A população alvo do presente trabalho foi constituída por membros da rede social de 168 pacientes de hanseníase (57 casos e 111 controles), dentre os 183 (64 casos e 119 controles) que participaram do estudo de caso-controle.

Os pacientes foram considerados "casos" na presença de incapacidade ou lesões precursoras de incapacidade, e "controles" na ausência destas. A classificação das incapacidades seguiu os critérios da Organização Mundial da Saúde (OMS) (14). As lesões precursoras de incapacidade corresponderam a infiltrações na face ou região periorbicular, lesões cutâneas ao redor do olho, sinais de comprometimento avançado no trajeto do nervo (espessamento com dor provocada ou espontânea, lesão cutânea extensa com anestesia, anestesia de área extensa, etc.) e lesões causadas por infecção secundária $(15,16)$.
Os pacientes selecionados tinham entre 20 e 70 anos de idade, eram residentes do Recife e estavam se consultando pela primeira vez ou completando um processo diagnóstico iniciado anteriormente nos Serviços Estaduais de Dermatologia Sanitária do Centro Integrado de Saúde Amaury de Medeiros e Centro de Saúde Agamenon Magalhães, Unidades de Referência das $3^{\mathrm{a}}$, $4^{\mathrm{a}}$ e $6^{\mathrm{a}}$ Regiões PolíticoAdministrativas entre novembro de 1993 e julho de 1994. Foram excluídos os pacientes que estavam se reintegrando ao programa após abandono ou alta por cura, os pacientes transferidos de outras unidades de saúde e os pacientes que não tinham iniciado o tratamento até o término do período de amostragem.

Até abril de 1994, 115 pacientes de hanseníase foram engajados no estudo. Destes, 12 não contaram com apoio durante a trajetória até o diagnóstico e 103 mencionaram ter recebido apoio. Destes últimos, nove pacientes foram excluídos porque não tinham iniciado o tratamento até o final do referido mês e 94 receberam solicitação para indicar um ou mais membros da rede social que pudessem ser entrevistados. Cinco pacientes não deram permissão para a realização de entrevistas. Os membros indicados da rede social de seis pacientes não foram entrevistados por motivos de viagem, doença e dificuldades operacionais para acesso.

A amostra do estudo foi constituída, portanto, por 93 membros da rede social de 83 pacientes de hanseníase (49,4\% dos 168 pacientes da populaçãoalvo que contaram com apoio de rede social). Entre os sujeitos selecionados, 34 eram membros da rede social de 30 casos e 59 eram membros da rede social de 53 controles. Os dados foram coletados nos domicílios e serviços de saúde, através de entrevistas semi-estruturadas executadas por uma enfermeira e uma assistente social, cuidadosamente treinadas, responsáveis também pelas entrevistas com os pacientes.

Um instrumento foi construído e ajustado para coletar informações junto aos membros da rede social sobre a) características sociodemográficas, como idade, sexo, história migra- 
tória, escolaridade, ocupação, acesso a meios de comunicação; b) manejo habitual de problemas de saúde; c) acesso a serviços de saúde; d) opiniões pessoais sobre as manifestações corporais do paciente, por exemplo, suspeitas, forma como adquiriu a doença, antevisão das conseqüências, expectativa de cura, modificações observadas na vida do paciente; e) participação no curso de ação até o diagnóstico, características da ajuda prestada e orientações fornecidas; f) contato prévio e informação sobre hanseníase, por exemplo, como se adoece, conseqüências futuras, expectativa de cura, fontes de informação. Um controle de qualidade sistemático foi efetuado. Os registros obtidos a partir das entrevistas com cada membro da rede social foram pré-analisados para assegurar a integridade, legibilidade e consistência interna das entrevistas.

Três subarquivos foram gerados usando o programa EPI-INFO. Depois de limpos e ordenados, esses subarquivos foram transformados em um grupo de variáveis recodificadas. A análise objetivou: a) detectar as variáveis capazes de discriminar os membros da rede de apoio dos pacientes de hanseníase classificados como casos ou controles; b) estabelecer a probabilidade dos sujeitos selecionados serem membros da rede social de casos ou controles usando análise de proporções e c) controlar a influência do contato prévio com hansenianos nas opiniões emitidas pelos membros da rede social. A significância da associação foi determinada usando a prova do qui quadrado $\left(\chi^{2}\right)$ ou o teste exato de Fisher (quando necessário). O termo "tendências" foi utilizado para descrever resultados em que se delineiam diferenças sem significância estatística.

\section{RESULTADOS}

A rede social dos pacientes de hanseníase em estudo era composta por uma alta proporção de mulheres, $85,3 \%$ entre os casos e $79,9 \%$ entre os controles. Em ambos os grupos, os membros da rede social viveram prati- camente toda a sua vida em áreas urbanas e tinham a mesma mediana de idade (43 anos com intervalo entre o primeiro e o terceiro quartis de 36 a 46 anos para a rede dos casos e de 29 a 52 anos para a rede dos controles). $\mathrm{O}$ acesso aos meios de comunicação era similar para os dois grupos e do total da rede social de casos e controles $94,6 \%$ ouviam rádio, $94,6 \%$ viam televisão, 69,9\% liam jornal e 47,3\% liam revistas. Os membros da rede social dos controles apresentaram escolaridade maior do que os membros da rede dos casos: mediana de 8 anos, com intervalo entre o primeiro e terceiro quartis de 4 a 11 anos, comparada à mediana de 5 anos de escolaridade e intervalo de 3 a 8 anos observados entre os membros da rede dos casos $(P=0,087)$.

$O$ perfil de ocupação da amostra revelou que $50 \%$ dos componentes da rede dos casos e $36,2 \%$ da rede dos controles exerciam atividades domésticas. Atividades não-manuais, como funções de escritório ou administrativas, eram desenvolvidas por $20,6 \%$ da rede social dos casos e $31 \%$ da rede dos controles.

A composição da rede de apoio de ambos os grupos apresentou um predomínio de familiares $(75,0 \%)$. Entre os controles, uma discreta tendência foi constatada para que este familiar fosse o cônjuge do paciente (37,9\% comparados com 23,5\% dos casos). Uma proporção significativamente maior dos membros da rede dos controles residia no mesmo domicílio do

TABELA 1. Contato prévio com hanseníase entre membros da rede social de pacientes no Recife, 1994

\begin{tabular}{|c|c|c|c|c|c|c|}
\hline \multirow[b]{3}{*}{ Variável } & \multirow[b]{3}{*}{ Categoria } & \multicolumn{4}{|c|}{ Membros da rede social } & \multirow[b]{3}{*}{$P$} \\
\hline & & \multicolumn{2}{|c|}{ Casos } & \multicolumn{2}{|c|}{ Controles } & \\
\hline & & No. & $\%$ & No. & $\%$ & \\
\hline $\begin{array}{l}\text { Tiveram contato prévio com } \\
\text { hanseníase }\end{array}$ & $\begin{array}{l}\text { Não } \\
\text { Familiar } \\
\text { Outro }\end{array}$ & $\begin{array}{r}20 / 34 \\
2 / 34 \\
12 / 34\end{array}$ & $\begin{array}{r}58,8 \\
5,9 \\
35,3\end{array}$ & $\begin{array}{r}33 / 59 \\
5 / 59 \\
21 / 59\end{array}$ & $\begin{array}{r}55,9 \\
8,5 \\
35,6\end{array}$ & 0,894 \\
\hline $\begin{array}{l}\text { Reação estigmatizante no primeiro } \\
\text { contato com hanseniano }\end{array}$ & $\begin{array}{l}\text { Sim } \\
\text { Não }\end{array}$ & $\begin{array}{l}6 / 13 \\
7 / 13\end{array}$ & $\begin{array}{l}46,1 \\
53,9\end{array}$ & $\begin{array}{r}9 / 23 \\
14 / 23\end{array}$ & $\begin{array}{l}39,1 \\
60,9\end{array}$ & 0,685 \\
\hline
\end{tabular}

paciente $(62,7 \%$ comparados com $38,2 \%$ da rede dos casos, $P=0,023)$.

$\mathrm{O}$ estudo mostrou que membros de ambas as redes sociais de apoio agem de forma similar quanto ao manejo de problemas de saúde e à utilização rotineira dos serviços de saúde. Do total dos entrevistados, a) $82,8 \%$ praticam automedicação e 66,7\% usam remédios caseiros; b) 69,9\% buscam atenção profissional somente após o insucesso do tratamento realizado por conta própria ou influenciado por familiares, amigos, etc.; c) 61,3\% utilizam comumente serviços de saúde públicos e filantrópicos; e d) 33,7\% se deslocam a pé até estes serviços. Constatou-se uma forte tendência, entre os membros da rede social dos controles $(46,6 \%$ comparados a $28,1 \%$ entre a rede dos casos, $P=0,077$ ), para mencionar a confiança no profissional como o motivo de escolha do serviço de saúde comumente utilizado.

A indagação sobre contato prévio com hanseníase (tabela 1) revelou semelhança entre os dois grupos: 58,8\% dos membros na rede social dos casos e $55,9 \%$ na dos controles nunca tinham tido contato prévio com a doença; 5,9\% na rede dos casos e 8,5\% na rede dos controles tiveram contato prévio com a doença pela existência de um familiar portador de hanseníase; e 35,3\% na rede dos casos e $35,6 \%$ na dos controles tiveram contato com a doença através de um conhecido com hanseníase. Entre os membros das duas redes sociais que já haviam entrado em contato com a doença, 41,7\% informaram 
que neste primeiro contato sentiram medo e rechaçaram o doente.

Quanto à disponibilidade de informação sobre hanseníase, $91,2 \%$ e $82,1 \%$ dos membros da rede social de casos e controles, respectivamente, não sabiam como se adoece. Entretanto, a maior parte dos entrevistados acredita que a hanseníase tem cura $(71,9 \%)$, menciona alguma possível conseqüência da doença $(83,9 \%)$ e tem acesso a fontes de informação sobre a hanseníase $(94,6 \%)$. Respectivamente, $45,5 \%$ e $52,5 \%$ dos componentes da rede social de casos e controles têm uma antevisão estigmatizante das conseqüências da hanseníase e citam paralisia, deformidade, mutilação e isolamento como prognósticos possíveis (tabela 2). Os membros das redes sociais que tiveram contato prévio com a doença não demonstraram ter mais informação do que aqueles que não tiveram contato prévio. As fontes de informação sobre hanseníase mais importantes mencionadas foram serviços de saúde $(22,8 \%)$, pessoa genérica $(26,4 \%)$, meios de comunicação de massa $(28,7 \%)$ e relações pessoais $(29,3 \%)$.

A tabela 3 compara as opiniões pessoais dos membros da rede social de casos e controles sobre a doença do paciente. Padrões similares foram identificados para ambos os grupos. Do total dos entrevistados, 59,1\% dizem saber como o paciente adquiriu a doença, entretanto $85,5 \%$ destes expressam uma opinião inadequada sobre este aspecto; $92,1 \%$ têm expectativas positivas em relação à cura; $41,1 \%$ acham que a doença afetou a vida do paciente. As suspeitas causadas pelo surgimento de sinais corporais dos pacientes foram de dermatomicose (41,9\% dos entrevistados), hanseníase $(27,2 \%)$ e câncer de pele $(11,1 \%)$. Outras doenças, como reumatismo, artrite, doenças da coluna e hipertensão, foram mencionadas. Observou-se, entre os membros da rede de apoio dos casos, uma forte tendência para considerar que a hanseníase modificou as relações pessoais destes pacientes.

A suspeita de hanseníase foi mais freqüente entre aqueles membros das redes sociais que haviam tido contato prévio com a doença $(80,9 \%$ comparados a 19,1\% entre os que não haviam tido contato prévio). As demais opiniões sobre a doença foram semelhantes, independente de experiência prévia com hanseníase.

A tabela 4 mostra que não foram encontradas diferenças entre os membros das redes sociais de ambos os grupos em relação à participação no processo diagnóstico. A intervenção mais freqüente foi a sugestão de buscar atenção profissional (86\% do total

TABELA 2. Informações sobre hanseníase entre membros da rede social de pacientes no Recife, 1994

\begin{tabular}{|c|c|c|c|c|c|c|}
\hline \multirow[b]{3}{*}{ Variável } & \multirow[b]{3}{*}{ Categoria } & \multicolumn{4}{|c|}{ Membros da rede social } & \multirow[b]{3}{*}{$P$} \\
\hline & & \multicolumn{2}{|c|}{ Casos } & \multicolumn{2}{|c|}{ Controles } & \\
\hline & & No. & $\%$ & No. & $\%$ & \\
\hline $\begin{array}{l}\text { Dispõe de alguma informação } \\
\text { sobre como adquirir } \\
\text { hanseníase }\end{array}$ & $\begin{array}{l}\text { Sim } \\
\text { Não }\end{array}$ & $\begin{array}{r}3 / 34 \\
31 / 34\end{array}$ & $\begin{array}{r}8,8 \\
91,2\end{array}$ & $\begin{array}{l}10 / 56 \\
46 / 56\end{array}$ & $\begin{array}{l}17,9 \\
82,1\end{array}$ & 0,356 \\
\hline Expectativa de cura & $\begin{array}{l}\text { Sim } \\
\text { Não }\end{array}$ & $\begin{array}{l}20 / 32 \\
12 / 32\end{array}$ & $\begin{array}{l}62,5 \\
37,5\end{array}$ & $\begin{array}{l}44 / 57 \\
13 / 57\end{array}$ & $\begin{array}{l}77,2 \\
22,8\end{array}$ & 0,141 \\
\hline $\begin{array}{l}\text { Tem alguma antevisão das } \\
\text { conseqüências }\end{array}$ & $\begin{array}{l}\text { Sim } \\
\text { Não }\end{array}$ & $\begin{array}{r}28 / 34 \\
6 / 34\end{array}$ & $\begin{array}{l}82,4 \\
17,6\end{array}$ & $\begin{array}{r}50 / 59 \\
9 / 59\end{array}$ & $\begin{array}{l}84,7 \\
15,3\end{array}$ & 0,764 \\
\hline $\begin{array}{l}\text { Antevisão estigmatizante das } \\
\text { conseqüências }\end{array}$ & $\begin{array}{l}\text { Sim } \\
\text { Não }\end{array}$ & $\begin{array}{l}15 / 33 \\
18 / 33\end{array}$ & $\begin{array}{l}45,5 \\
54,5\end{array}$ & $\begin{array}{l}31 / 59 \\
28 / 59\end{array}$ & $\begin{array}{l}52,5 \\
47,5\end{array}$ & 0,517 \\
\hline $\begin{array}{l}\text { Dispõe de fonte de informação } \\
\text { sobre hanseníase }\end{array}$ & $\begin{array}{l}\text { Sim } \\
\text { Não }\end{array}$ & $\begin{array}{r}31 / 34 \\
3 / 34\end{array}$ & $\begin{array}{r}91,2 \\
8,8\end{array}$ & $\begin{array}{r}56 / 58 \\
2 / 58\end{array}$ & $\begin{array}{r}96,6 \\
3,4\end{array}$ & $0,354^{a}$ \\
\hline
\end{tabular}

aTeste exato de Fisher. dos entrevistados). Nas outras categorias (acompanhar até o serviço de saúde, marcar consulta, indicar drogas, indicar remédios caseiros e sugerir que a doença fosse mantida em segredo) a maioria dos membros de ambos os grupos não teve participação ativa. Entre os membros da rede dos casos não houve indicação para o uso de remédios caseiros.

Tanto os pacientes quanto os membros das suas redes sociais de apoio revelaram uma expectativa positiva de cura e concordam sobre o fato de que a hanseníase afeta a vida dos seus portadores, particularmente modificando as suas relações pessoais. Ademais, tanto pacientes como membros de sua rede social apresentaram uma antevisão estigmatizante da hanseníase, mais acentuada entre os membros das redes de apoio, os quais, entretanto, formularam um prognóstico mais positivo para o paciente.

O autodiagnóstico de hanseníase foi pouco influenciado pelos membros da rede social $(17,3 \%)$. No entanto, observou-se uma tendência entre os controles para admitir esta influência $(22,0 \%$ dos controles contra $8,0 \%$ dos casos, $P=0,115)$ e informar que a sugestão ocorreu antes da primeira consulta médica. Entre o grupo dos controles, $89,1 \%$ dos pacientes solicitaram ajuda a sua rede de apoio. A participação da rede social nas decisões relacionadas com a busca de atenção profissional foi importante ou fundamental para 79,2\% dos controles e para $96,6 \%$ dos casos.

Não foram identificadas diferenças entre os pacientes dos dois grupos quanto à correspondência entre a rede social que participou do curso de ação durante o processo de diagnóstico e a rede social habitualmente mobilizada. Do total dos pacientes, $10,4 \%$ informaram que a rede disponível na doença foi diferente da habitual; para 71,4\% a correspondência foi parcial; e para $18,2 \%$ a correspondência foi total.

\section{DISCUSSÃO E CONCLUSÕES}

Os resultados revelam um perfil homogêneo para os membros da rede social de apoio dos pacientes de hanse- 
TABELA 3. Opinião pessoal sobre a hanseníase entre membros da rede social de pacientes no Recife, 1994

\begin{tabular}{|c|c|c|c|c|c|c|}
\hline \multirow[b]{2}{*}{ Variável } & \multirow[b]{2}{*}{ Categoria } & \multicolumn{4}{|c|}{ Membros da rede social } & \multirow[b]{2}{*}{$P$} \\
\hline & & No. & $\%$ & No. & $\%$ & \\
\hline Diz saber como o paciente adquiriu a doença & $\begin{array}{l}\text { Sim } \\
\text { Não }\end{array}$ & $\begin{array}{l}21 / 34 \\
13 / 34\end{array}$ & $\begin{array}{l}61,8 \\
38,2\end{array}$ & $\begin{array}{l}34 / 59 \\
25 / 59\end{array}$ & $\begin{array}{l}57,6 \\
42,4\end{array}$ & 0,697 \\
\hline $\begin{array}{l}\text { Dispõe de opinião adequada sobre como o } \\
\text { paciente adquiriu a doença }\end{array}$ & $\begin{array}{l}\text { Sim } \\
\text { Não }\end{array}$ & $\begin{array}{r}2 / 21 \\
19 / 21\end{array}$ & $\begin{array}{r}9,5 \\
90,5\end{array}$ & $\begin{array}{r}6 / 34 \\
28 / 34\end{array}$ & $\begin{array}{l}17,6 \\
82,3\end{array}$ & $0,696^{a}$ \\
\hline $\begin{array}{l}\text { Acha que a hanseníase pode ter } \\
\text { conseqüências estigmatizantes }\end{array}$ & $\begin{array}{l}\text { Sim } \\
\text { Não }\end{array}$ & $\begin{array}{r}8 / 33 \\
25 / 33\end{array}$ & $\begin{array}{l}24,2 \\
75,8\end{array}$ & $\begin{array}{r}7 / 58 \\
51 / 58\end{array}$ & $\begin{array}{l}12,1 \\
87,9\end{array}$ & 0,134 \\
\hline $\begin{array}{l}\text { Acha que a doença afetou as relações } \\
\text { pessoais do paciente }\end{array}$ & $\begin{array}{l}\text { Sim } \\
\text { Não }\end{array}$ & $\begin{array}{r}1 / 13 \\
12 / 13\end{array}$ & $\begin{array}{r}7,7 \\
92,3\end{array}$ & $\begin{array}{r}9 / 24 \\
15 / 24\end{array}$ & $\begin{array}{l}37,5 \\
62,5\end{array}$ & $0,065^{a}$ \\
\hline
\end{tabular}

aTeste exato de Fisher.

níase, quando são consideradas as características sociodemográficas e de acesso aos meios de comunicação. Na rede social mobilizada pelos controles observou-se uma forte tendência para maior escolaridade.

A grande maioria dos membros das redes sociais dos portadores de hanseníase no presente estudo não sabem como a doença é adquirida, embora uma proporção importante acredite em cura. Este fato sugere que na evolução sociocultural das construções sobre a hanseníase a incurabilidade passou a ter um menor peso. Mesmo assim, os modelos culturais e sociais mencionados pelos membros das redes de apoio traduzem uma antevisão estigmatizante das conseqüências da hanseníase (17), o que sugere que a reação social espontânea diante da doença se mantém intimamente relacionada com uma imagem negativa.

No Brasil, em 1988, uma campanha nacional de estímulo ao diagnóstico precoce da hanseníase, realizada através dos meios de comunicação de massa, particularmente a televisão, divulgou as principais manifestações corporais da doença e a disponibilidade de drogas eficazes para o seu tra- tamento. No primeiro trimestre de 1994, durante o trabalho de campo desta pesquisa, a televisão divulgou, em nível local e de forma esporádica, as mesmas informações. O material informativo distribuído nos serviços de saúde enfatiza que a hanseníase tem cura.
Aproximadamente metade dos membros das redes sociais dos pacientes que participaram do presente estudo mencionaram os meios de comunicação de massa e os serviços de saúde como fontes de informação sobre hanseníase. Contudo, a divulgação através da mídia de descobertas

TABELA 4. Participação dos membros da rede social no curso de ação que levou ao diagnóstico de pacientes de hanseníase no Recife, 1994

\begin{tabular}{|c|c|c|c|c|c|c|}
\hline \multirow[b]{3}{*}{ Variável } & \multirow[b]{3}{*}{ Categoria } & \multicolumn{4}{|c|}{ Membros da rede social } & \multirow[b]{3}{*}{$P$} \\
\hline & & \multicolumn{2}{|c|}{ Casos } & \multicolumn{2}{|c|}{ Controles } & \\
\hline & & No. & $\%$ & No. & $\%$ & \\
\hline $\begin{array}{l}\text { Sugeriu buscar atenção } \\
\text { profissional }\end{array}$ & $\begin{array}{l}\text { Sim } \\
\text { Não }\end{array}$ & $\begin{array}{r}31 / 34 \\
3 / 34\end{array}$ & $\begin{array}{r}91,2 \\
8,8\end{array}$ & $\begin{array}{l}49 / 59 \\
10 / 59\end{array}$ & $\begin{array}{l}83,0 \\
17,0\end{array}$ & $0,361^{a}$ \\
\hline $\begin{array}{l}\text { Acompanhou o paciente até o } \\
\text { serviço de saúde }\end{array}$ & $\begin{array}{l}\text { Sim } \\
\text { Não }\end{array}$ & $\begin{array}{r}7 / 34 \\
27 / 34\end{array}$ & $\begin{array}{l}20,6 \\
79,4\end{array}$ & $\begin{array}{l}17 / 59 \\
42 / 59\end{array}$ & $\begin{array}{l}28,8 \\
71,2\end{array}$ & 0,385 \\
\hline $\begin{array}{l}\text { Marcou consulta para o } \\
\text { paciente }\end{array}$ & $\begin{array}{l}\text { Sim } \\
\text { Não }\end{array}$ & $\begin{array}{r}2 / 34 \\
32 / 34\end{array}$ & $\begin{array}{r}5,9 \\
94,1\end{array}$ & $\begin{array}{r}8 / 59 \\
51 / 59\end{array}$ & $\begin{array}{l}13,5 \\
86,5\end{array}$ & $0,361^{a}$ \\
\hline Indicou drogas & $\begin{array}{l}\text { Sim } \\
\text { Não }\end{array}$ & $\begin{array}{r}2 / 34 \\
32 / 34\end{array}$ & $\begin{array}{r}5,9 \\
94,1\end{array}$ & $\begin{array}{r}4 / 59 \\
55 / 59\end{array}$ & $\begin{array}{r}6,8 \\
93,2\end{array}$ & $1,000^{a}$ \\
\hline Indicou remédios caseiros & $\begin{array}{l}\text { Sim } \\
\text { Não }\end{array}$ & $\begin{array}{r}0 / 34 \\
34 / 34\end{array}$ & $100, \overline{0}$ & $\begin{array}{r}4 / 59 \\
55 / 59\end{array}$ & $\begin{array}{r}6,8 \\
93,2\end{array}$ & $0,297^{a}$ \\
\hline $\begin{array}{l}\text { Sugeriu manter doença em } \\
\text { segredo }\end{array}$ & $\begin{array}{l}\text { Sim } \\
\text { Não }\end{array}$ & $\begin{array}{r}3 / 34 \\
31 / 34\end{array}$ & $\begin{array}{r}8,8 \\
91,2\end{array}$ & $\begin{array}{r}4 / 59 \\
55 / 59\end{array}$ & $\begin{array}{r}6,8 \\
93,2\end{array}$ & $0,703^{a}$ \\
\hline
\end{tabular}

Teste exato de Fisher. 
científicas e soluções tecnológicas para diferentes problemas de saúde poderia ser outra importante razão para estas expectativas.

No âmbito técnico, se busca estabelecer uma distinção básica entre a realidade epidemiológica hanseníase, com diagnóstico refinado e disponibilidade de tratamento com drogas eficazes (18), e a lepra como uma representação social do passado, a quem se atribuem efeitos que pertencem a outras entidades epidemiológicas (19). Como a educação da população baseou-se na deformidade e na mutilação e fortalece o temor do contágio, observa-se a persistência de um hiato entre a concepção institucionalizada sobre a doença e a concepção das pessoas comuns, que continuam atuando em função das metáforas recebidas (20). É importante considerar que o medo do contágio pressupõe medo de suas conseqüências; portanto, é a concepção sobre quais são as conseqüências físicas e sociais da hanseníase que constitui o elemento fundamental a ser trabalhado na luta pela eliminação desta doença.
A mobilização espontânea dos membros da rede social por pacientes de hanseníase durante o processo que levou ao diagnóstico e a influência da rede social nas decisões relacionadas com a busca de atenção profissional demonstram a importância deste apoio. A rede social estimulou uma maior valorização das manifestações corporais, na ausência de uma suspeita de hanseníase ou até mesmo na tentativa de negá-la. Ainda assim, a rede social teve pouca participação na formulação de um autodiagnóstico de hanseníase.

Apenas uma quarta parte dos membros das redes sociais suspeitou, em algum momento, que o paciente era portador de hanseníase. A baixa percepção do risco de hanseníase reforça a concepção da invisibilidade de suas manifestações corporais. Esta dificuldade para suspeitar da ocorrência de hanseníase diante dos sinais e sintomas da doença foi observada, também, entre os pacientes e os profissionais de saúde entrevistados durante a avaliação dos serviços de saúde. Estes resultados sugerem que a atitude corrente em relação à hanseníase pode facilitar a propagação da doença e a instalação ou agravamento das suas conseqüências físicas e sociais.

$\mathrm{O}$ apoio social recebido pelos pacientes de hanseníase antes do diagnóstico não é um indicativo da disponibilidade de apoio após a confirmação da doença. A hanseníase continua sendo mais temida do que outras doenças, inclusive fatais. Entre os membros das redes sociais que mencionam contato prévio com esta doença, uma grande proporção admitiu reações de medo e rechaço em relação à pessoa doente. Este contexto aponta para a existência de um quadro transicional onde se confrontam uma expectativa positiva de cura e uma visão estigmatizante sobre as conseqüências da hanseníase. Assim, é relevante estimular a indagação sobre a reação dos membros da rede social depois de confirmado o diagnóstico da doença.

Agradecimentos. Agradecemos à assistente social Suzette Brito e à enfermeira Cácia Mendes pela participação no trabalho de campo.

\section{REFERÊNCIAS}

1. Slovic P. Perception of risk. Science 1987;236: 280-285.

2. Wilson R, Crouch EAC. Risk assessment and comparison: an introduction. Science 1987;236: 267-270.

3. Minayo MC de S. Abordagem antropológica para avaliação de políticas sociais. Rev Saude Publica 1991;25(3):233-238.

4. Oliven RG. Pesquisas antropológicas no contexto urbano. Em: Antropologia de grupos urbanos. $2^{\text {a }}$ ed. Petrópolis: Editora Vozes; 1987 : 21-47.

5. Infante-Castañeda C. Bases para el estudio de la interacción familia-redes sociales-uso de servicios de salud. Salud Publica Mex 1988;30: 175-196.

6. Broadhead WE, Kaplan BH, James SA, Wagner EH, Schoenbach VJ, Grimson R, et al. The epidemiologic evidence for a relationship between social support and health. Am J Epidemiol 1983;117(5):521-537.

7. Kaufmann A, Mariam SG, Neville J. The social dimension of leprosy. London: ILEP; 1986.

8. Berkman LF. Social networks, support and health: taking the next step forward. Am J Epidemiol 1986;123(4):559-562.
9. Valencia LB, Ventura ER, Paz SG. Managing triadic interaction: a guide to Hansen's control. Geneva: UNDP/World Bank/WHO; 1988. (TDR, Social and Economic Research Project, Report No. 3).

10. Haguette TMF. Metodologias qualitativas na sociologia. Rio de Janeiro: Editora Vozes; 1988.

11. Kleinman A. The illness narratives: suffering, healing, and the human condition. New York: Basic Books; 1988.

12. Minayo MC de S. O desafio do conhecimento: pesquisas qualitativas em saúde. Rio de Janeiro: Hucitec-Abrasco; 1992.

13. Habermas J. Ações, atos de fala, interações mediadas pela linguagem e mundo da vida. Em: Habermas J, ed. Pensamento pós-metafísico. Rio de Janeiro: Tempo Brasileiro; 1990: 65-104.

14. World Health Organization. A guide to leprosy control. 2nd ed. Geneva: WHO; 1988.

15. Brand PW, Fritschi EP. Rehabilitation in leprosy. Em: Hastings RC, ed. Leprosy. New York: Churchill Livingstone; 1985:287-319.

16. Bryceson A, Pfaltzgraff RE. Leprosy. 2nd ed. New York: Churchill Livingstone; 1979 (reprinted 1980).
17 Lessa ZL. Doença e estigma: cadernos de história e saúde no 2. Casa de Oswaldo Cruz; 1992

18. Srinivasan H. Newer tasks for leprosy works. Indian J Lepr 1990;62(4):1-5.

19. Brasil, Ministério da Saúde. Controle da hanseníase: uma proposta de integração ensinoserviço. Rio de Janeiro: Secretaria especial de programas especiais de saúde, Divisão Nacional de Dermatologia Sanitária/NUTES; 1989.

20. Sevilla E. Evolución de las representaciones sociales de la lepra en Colombia. Em: Los mutilados del oprobio. Santa Fe de Bogotá: Colcultura/Tercer Mundo Editores; 1995: 51-69.

Manuscrito recebido em 21 de julho de 1995. Aceito em versão revisada em 1 de dezembro de 1995. 
ABSTRACT This article describes a study done in Recife, Brazil, between November 1993 and July 1994 to explore the opinions of the members of the social network (for example, family members, friends, and neighbors) of carriers of Hansen's disease regarding their estimation, interpretation, and management of physical manifestations of the disease in the time leading up to diagnosis. The sample consisted of 93 members of the social network, ranging in age between 20 and 70 years, who supported the course of action of 83 patients diagnosed in the study period. The analysis sought to detect differing capacities among the members of the patients' social network to discriminate between persons classified as cases (presence of disabilities or precursor lesions) or controls.

The study found a lack of information about transmission of Hansen's disease and revealed a transitional phase in which there was expectation of cure along with a stigmatizing view of the consequences of the disease. Only one-quarter of the study subjects suspected prior to diagnosis that the patient had Hansen's disease, which suggests low perception of the risk represented by the disease and reinforces the idea that its physical manifestations can be invisible. The results reveal a profile of perception and management of Hansen's disease that favors its propagation and the development or worsening of its physical and social consequences.

\section{Congreso Internacional sobre Enfermedades de Transmisión Sexual}

Fechas: $\quad 19$ a 22 de octubre de 1997

Lugar: $\quad$ Sevilla, España

La Sociedad Internacional para la Investigación de las Enfermedades de Transmisión Sexual (ISSTDR) y la Unión Internacional contra las Enfermedades Venéreas y las Treponematosis (IUVDT) se complacen en convocar a médicos, investigadores, y otros profesionales de la salud y sus campos afines a este foro de discusión, donde se presentarán los resultados de las investigaciones más recientes sobre numerosos aspectos de las enfermedades de transmisión sexual, especialmente la infección por VIH y el sida. Habrá simposios, conferencias, talleres, carteles y grupos de discusión. El inglés será el idioma oficial del Congreso, pero habrá traducción al español en las sesiones plenarias. La fecha límite para el recibo de resúmenes de investigaciones es el 30 de abril de 1997.

Información para América Latina:

Secretaría del Congreso Internacional

de Enfermedades de Transmisión Sexual

Apartado 6077

41080 Sevilla, España

Fax: (34) 54377413

Información para Estados Unidos y Canadá:

ISSTDR/HCC

One Bridge Plaza

Suite 350

Fort Lee, New Jersey 07024-9885

Estados Unidos de América

Fax: (201) 947-8406 